\title{
Missing data on 3-month modified Rankin Scale may influence results of functional outcome after intravenous thrombolysis in observational studies
}

\author{
Stefano Forlivesi ${ }^{1}$ (D) Manuel Cappellari $^{1} \cdot$ Paolo Bovi $^{1}$
}

Published online: 23 June 2016

(c) Springer Science+Business Media New York 2016

\section{Dear Editor,}

The Safe Implementation of Treatments in Stroke (SITS)Thrombolysis is an internet-based registry, which currently includes more than one hundred thousand thrombolysed patients from about 1400 centers all over the world [1]. As of May 31 2016, $57.8 \%$ of the registered patients resulted functionally independent [modified Rankin Scale (mRS) $\leq 2]$ at 3 months after intravenous thrombolysis (IVT), but data on 3-month mRS were missing in a significant proportion $(24 \%)$ of the registered patients [2]. A recent analysis on 29,618 patients registered in the SITS-Thrombolysis reported functional independence at 3 months in $14,148 / 23,987$ (59\%) patients, and missing data on 3-month mRS in $5631(19 \%)$ patients [3]. On the other hand, a systematic review of randomized controlled trials (RCTs) on IVT reported a lower proportion of thrombolysed patients $(1611 / 3483,46.3 \%)$ who were functionally independent at the end of follow-up [4]. We speculate that missing data in observational studies are responsible of overestimation of the proportion of patients with 3-month $\mathrm{mRS} \leq 2$ after IVT. We analyzed data prospectively collected from 207 consecutive stroke patients treated with IVT at the Stroke Unit, Azienda Ospedaliera Universitaria Integrata Verona, from January 1 to December 31, 2015. Since a significant proportion of patients (29\%) did not attend the 3-month scheduled follow-up visit or telephonic interview, we went to the missing patients' residence to assess 3-month mRS and we were able to get data from $54 / 60(90 \%)$ missing patients. Of these patients only $20 / 54$

Stefano Forlivesi

forlivesi.sf@gmail.com

1 Stroke Unit, Azienda Ospedaliera Universitaria Integrata Verona, Piazzale Aristide Stefani 1, 37126 Verona, Italy
(37\%) had $\mathrm{mRS} \leq 2$, compared with 86/147 (58.5\%) functionally independent patients who attended the 3 -month regular follow-up $(\mathrm{p}=0.01)$. Overall proportion of patients with 3 -month $\mathrm{mRS} \leq 2$ was $52.7 \%$, which is slightly higher compared with RCTs [4], but lower compared with previously reported observational studies $[2,3]$. In conclusion, our findings suggest that missing data on 3-month mRS may influence results of functional outcome after IVT in observational studies.

Compliance with ethical standards

Conflicts of interest The authors declare no conflicts of interest.

\section{References}

1. SITS (Safe Implementation of Treatments in Stroke) International. SITS-Thrombolysis registry, http://www.sitsinternational.org/regis tries/sits-thrombolysis/. 31 May 2016

2. SITS (Safe Implementation of Treatments in Stroke) International. SITS database, https://db.sitsinternational.org/intranet/sitsdata base/sitscountry_IT/sitshospital_VER/. 31 May 2016

3. Ahmed N, Kellert L, Lees KR, Mikulik R, Tatlisumak T, Toni D (2013) Results of intravenous thrombolysis 4.5 to 6 hours and updated results within 3-4.5 hours of onset of acute ischemic stroke recorded in the Safe Implementation of Treatment in Stroke International Stroke Thrombolysis Register (SITS-ISTR): an observational study. JAMA Neurol 70:837-844

4. Wardlaw JM, Murray V, Berge E, Del Zoppo GJ (2014) Thrombolysis for acute ischaemic stroke. Cochrane Database Syst Rev 7(CD000213) 